\title{
SIRNA KNOCKDOWN SKP2 GENE IN BREAST CANCER
}

Wael S. Abdel-Mageed

\author{
Molecular Biology Department. Genetic Engineering and \\ Biotechnology Research Institute (GEBRI), Sadat \\ University, Sadat City, Minufiya, Egypt.
}

Received 16/11/2015- Accepted 20/12/2015

\begin{abstract}
The S-phase kinase-associated protein-2 (SKP2) plays a strategic role in ubiquitin-mediated proteolysis, which effects in the development of cells from inactivity to proliferative state. SKP2 is overexpressed in a variety of tumor. In this study, we used small interfering RNAs (siRNAs) to inhibit the SKP2 expression in Breast cancer cells preface investigate the role of SKP2 in breast tumorigenesis. Two Breast cancer cell lines (MCF-7 and T47D) were transfected with siRNAs targeted against SKP2. Our results showed one SKP2-siRNA specifically and efficiently reduced the levels of the SKP2 protein by $90 \% 48 \mathrm{~h}$ after transfection in MCF-7 cell line. In the transfected cells, p27 protein was accumulated inversely related to Skp2 siRNA transfected Breast cells. Skp2 siRNA inhibited the cell growth of breast cells in vitro. Moreover, Skp2 siRNA also suppressed tumor proliferation in vivo. Our results suggest that siRNA-mediated gene silencing of Skp2 can be a potent tool of cancer gene therapy for suppression of p27 degradation in breast cancer.
\end{abstract}

\section{INTRODUCTION}

Breast cancer is a common and complex disease often wanting multimodality care. It's may be treated with surgical resection, radiotherapy (RT), chemotherapy, hormonal therapy, and targeted therapies, or a combination thereof $[1,2]$. Molecular cancer therapeutics are also attracting care, and targeted molecular therapies that already contributed to effective new treatments for breast cancer [3]. Skp2 is oncogenic gene with high frequent amplification and

* Correspondence Author (e mail: : drwael.abdelmageed0@gmail.com) 
overexpression correlated with the grade of malignancy $[4,5]$. The $S$ phase kinase-associated protein2 (Skp2) a member of the F-box family of substrate-recognition subunits of Skp1-Cullin-F-box ubiquitin-protein ligase complexes, is necessary for $\mathrm{p} 27$ ubiquitination and degradation [6]. Skp2 is proposed to be an onco-protein because of its ability to trigger Lys 48 ubiquitination and degradation of p27 [7]. The proteolytic degradation of BRCA2 in PCa cells, suggesting that the BRCA2-Skp2 interaction may play a role in prostate tumorigenesis [8]. Skp2 overexpression plays an important role in the pathogenesis of colorectal cancer [9]. One of the key players regulating cell cycle progression is the F-box protein Skp2 [10]. Cell adhesion is essential for cell cycle progression in most normal cells. Loss of adhesion dependence is a hallmark of cellular transformation. The F-box protein Skp2 (S-phase kinase-associated protein 2) controls $G$ (1)-S-phase progression and is subject to adhesion-dependent transcriptional regulation. $[\mathbf{1 1}, \mathbf{1 2}] . \quad$ Skp2 is a subunit of the SCF ubiquitin protein ligase, which plays a vital role in the control of tumorigenesis via its regulation of G (1)-S transition. Deregulation of Skp2 in various types of cancers correlates with aggressive clinical behavior and poor prognosis $[\mathbf{1 3}, \mathbf{1 4}]$. MicroRNA (miRNA) inhibition is a promising biological strategy for breast cancer therapy. However this application is limited by the lack of efficient miRNA inhibitor delivery systems $[\mathbf{1 5}, \mathbf{1 6}]$. Therapeutics based on short interfering RNAs (siRNAs) has great potential to treat human diseases. However, the clinical application of siRNAs has been limited by their poor intracellular uptake, low serum stability, and inability to target specific cells [17-19]. In summary, S-phase kinase-associated protein 2 (Skp2) is an F box protein that plays critical roles in cell-cycle progression, overexpression of Skp2 is associated with poor prognosis and metastasis in many cancers and is a well validated drug target [20]. Skp2 is a promising target for restricting cancer stem cell and cancer progression [21, 22]. Skp2 may be a novel and attractive therapeutic target for cancers $[\mathbf{2 3}, \mathbf{2 4}]$.

\section{MATERIALS AND METHODS}

\section{siRNA preparation}

The mRNA sequences of SKP2 gene is submitted to siDirect2. It is an siRNA designing software available online.32, the siRNA molecules 
are designed using combined approach of Ui-Tei, Amarzguioui and Reynolds rules.33 The other parameter that was applied is melting temperature $(\mathrm{Tm})$ below $21.5^{\circ} \mathrm{C}$ for siRNA duplex. The validation of the predicted siRNA molecules was performed using an online tool called siRNA at whitehead. This gives a list of siRNA sequences for SKP2 and the best sequence is selected considering the melting temeperatures and GC content. These included siRNA1, which corresponds to positions $22-44$ of the SKP2 open reading frame; and siRNA2, which corresponds to positions 422-444. The sequences for silencing the GAPDH gene region were used as the control siRNA. All siRNAs were prepared using a transcription-based method with the silencer siRNA construction kit (Ambion) according to the manufacturer's instructions.

\section{Cell culture, transfections, and transfection efficiency Breast cancer cell lines}

MCF-7 and T47D obtained from Prof. Dr. Stefan Barth, Fraunhofer IME, RWTH Achen University, Germany. This cell line revealed high SKP2 protein expression level [25]. The cell were cultured in DMEM supplemented with $10 \% \mathrm{FBS}$ at $371 \mathrm{C}$ in $5 \% \mathrm{CO} 2$. At 1 day prior to transfection, cells were seeded at $1 \times 10^{6}$ cells per $25-\mathrm{cm}^{2}$ culture flasks and cells were plated in 6 -well plates at a seeding density of $5 \times 10^{5}$ cells and simultaneously transfected, alone or in combination with 40 nmol SKP-siRNAs (Ambion, TX, USA) and EGCG (Sigma-Aldrich, St. Louis, MO, USA). The siPORT NeoFX Transfection Agent (Invitrogenby Life Technologies) being used for siRNAs delivery and cells were cultured in Opti-MEM I (Gibco-Invitrogen, Paisley, UK) reduced serum medium. 96-well plates used and reduced the reagent volumes by one-tenth. All experiments being performed in triplicate. Cells were incubated for $4 \mathrm{~h}$ with siRNAs or PBS (control cells) in lipid transfection reagent. After this, one-third of the transfection volume of fresh culture medium with $10 \%$ FCS was added. Cells were harvested 24, 48, and $72 \mathrm{~h}$ after the transfection, protein expression was evaluated and the immunofluorescence assay and FACS analysis were performed. The Fluorescein siRNA Labeling Kit (Ambion) was used to label chemically synthesized siRNAs with Cy3.

Western blot analysis

Cells were lysed at a density of $5 \times 10^{4}$ cells $/ \mathrm{ml}$ in lysis buffer $(1 \%$

Triton X-100, $1 \mathrm{mM}$ EDTA, $150 \mathrm{mM} \mathrm{NaCl}$, and $50 \mathrm{mM}$ Tris- $\mathrm{HCl})$ on ice for $10 \mathrm{~min}$. Equal amounts of lysate were separated by SDS- 
PAGE on an acrylamide gel. Proteins were transferred to a nitrocellulose membrane (Pierce Biotechnology, Inc., Rockford, IL, USA) and incubated with antibodies specific to SKP2, p27 and $\beta$ actin (Santa Cruz Biotechnology, Inc., Santa Cruz, CA, USA). The membranes were then incubated with the appropriate secondary antimouse or anti-rabbit horseradish peroxidase-conjugated antibodies (Bio-Rad, Inc., Hercules, CA, USA) for $2 \mathrm{~h}$. Immuno-complexes were detected using an enhanced chemiluminescence reagent (Amersham Pharmacia Biotech, Buckinghamshire, UK). An anti-b-actin blot was created in parallel as a loading control.

\section{Statistical analysis}

All the experiments were repeated at least three times with each treatment performed in triplicate. The b-actin signal was standard measure on Western blots, along with 95\% confidence intervals, were calculated. The results were analyzed using the General Linear Models of Statistica (StatSoft, Inc., Tulsa, OK, USA).

\section{Acknowledgements}

I would like to thank Prof. Dr. Stefan Barth, Fraunfofer IME, RWTH Achen University, Germany for supporting this work.

\section{RESULTS}

\section{Specific inhibition of SKP2 expression by siRNAs}

We first determined whether the endogenous level of SKP2 protein in the MCF-7 breast cancer cells could be inhibited by siRNAs. The transfection efficiency of the siRNAs was $>90 \%$ at a concentration of $40 \mathrm{nM}$ for each cell line after $24 \mathrm{~h}$ of transfection. Transfection of the MCF-7 cells with tow SKP2-specific siRNAs (siRNA1 and siRNA2) at a concentration of $40 \mathrm{nM}$ showed reduced the level of SKP2 protein by $15-30 \%$ after $24 \mathrm{~h}$ of transfection and resulted in a statistically significant reduction in the SKP2 protein level by 65-90\% 48 and 72 $\mathrm{h}$ after transfection with siRNA1. In contrast, SKP2 protein levels remained unchanged in the siRNA2, b-actin and PBS (Figure 1). 


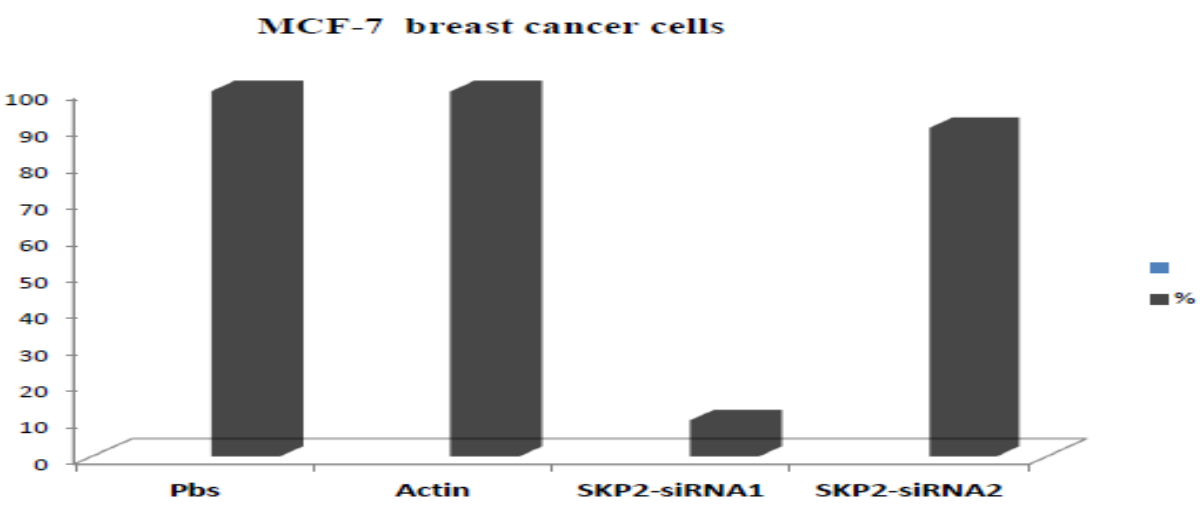

Figure (1): siRNAs against SKP2 specifically inhibit its expression.

MCF-7 breast cancer cells were transfected with SKP2-siRNAs 1-2 and PBS. The histogram shows the inhibition rate of SKP2 production. The same filter was also investigated with b-actin antibody to control for even loading.

\section{Reduction of SKP2 resulted in elevated p27 protein level}

Skp2 is proposed to be an onco-protein because of its ability to trigger Lys 48 ubiquitination and degradation of p27 [7]. Consistent with the Western blot findings (Figure 2a,b), siRNA1 reduced the number of fluorescent cells by up to $90 \%$, with a maximal effect $48 \mathrm{~h}$ after transfection (Figure 3). Different observations at diverse time were made in T47D cells (Figure 4). Furthermore, the SKP2 protein reduction occurred in a dose-dependent manner from 1 to $40 \mathrm{nM}$. At 40nM, siRNA had a significant inhibiting effect on the production of SKP2 protein $(\mathrm{P}<0.005)$, but there was no statistically significant difference in the reduction in the SKP2 protein level between 40 and $90 \mathrm{nM}(\mathrm{P}>0.005)$. SKP2 production was inhibited by $90 \%$ using 90 nM siRNA1. These data suggest that siRNAs can efficiently and specifically inhibit the expression of the target gene in breast cancer cells. The level of the p27 protein was examined in the breast cell lines transfected with siRNAs. The level of the p27 protein was increased in the transfected MCF-7 cells, and the level was inversely proportional to the level of SKP2. In contrast, there was no change in p27 production in the T47D cells even though SKP2 expression was dramatically reduced in the cells after transfection (Figure 4). 


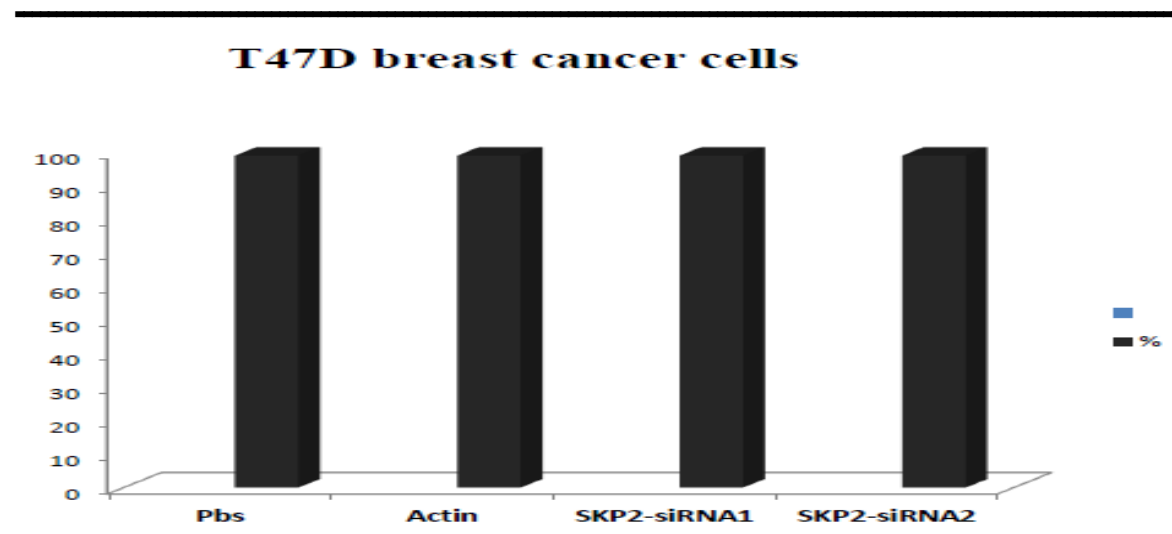

Figure (4): siRNAs against SKP2 specifically inhibit its expression.

T47D breast cancer cells were transfected with SKP2-siRNAs 1-2 and PBS. The histogram shows no significance in inhibition of SKP2 production. The same filter was also investigated with b-actin antibody to control for even loading.

\section{Transfection efficiency}

The evaluation of new siRNA sequences requires efficient and reliable transfection protocols to achieve the strongest gene silencing effects. Many protocols and reagents have been developed for the transfection of siRNAs into eukaryotic cell lines. Transfection efficiencies were determined by flow cytometry and fluorescence microscopy using FITC-labeled siRNAs transfected at a concentration of $200 \mathrm{nM}$ with different transfection protocols.

MCF-7 breast cancer cells
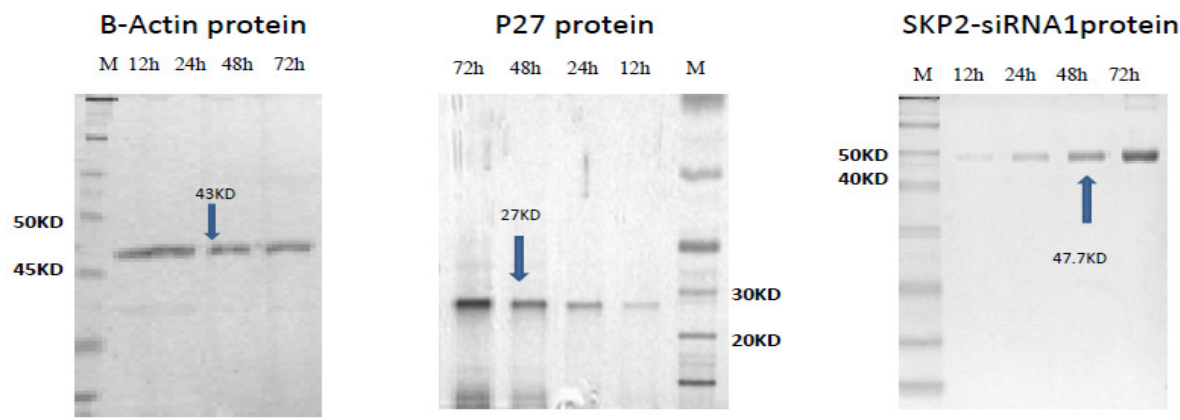

Figure (2a): Effect of SKP2 reduction on p27 expression.

MCF-7 breast cancer lines were transfected with SKP2-siRNA1. Western blot analysis of SKP2 and p27 protein expression was performed after transfection. 


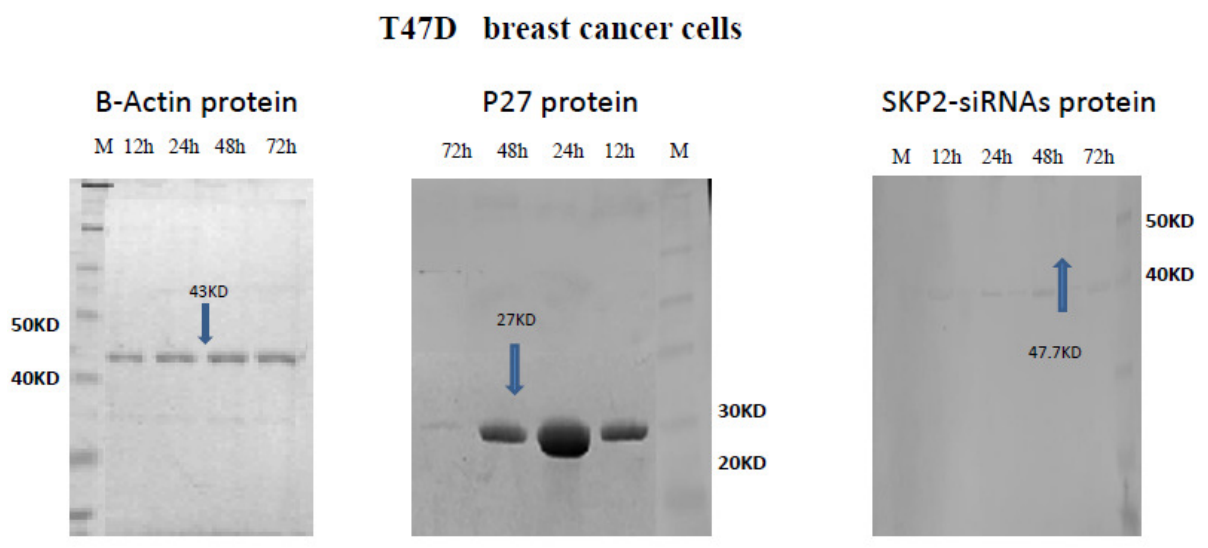

Figure (2b): Effect of SKP2 reduction on p27 expression.

T47D breast cancer lines were transfected with SKP2-siRNA1-2. Western blot analysis of SKP2 and p27 protein expression effects for siRNAs and on the opposite side there is expression with p27 protein after transfection.

After 12-h incubation, cells were washed twice and the relative amount of shifted cells in channel FL-1 in G1 was monitored via flow cytometry. Figure 5 shows the transfection efficiency when $200 \mathrm{nM}$ FITC-labeled siRNA was introduced into MCF-7 and T4D7 cells using the lipofection solution Hiperfect (Qiagen). After 12-h incubation, more than $90 \%$ of both cell lines were stained (Figure 5).

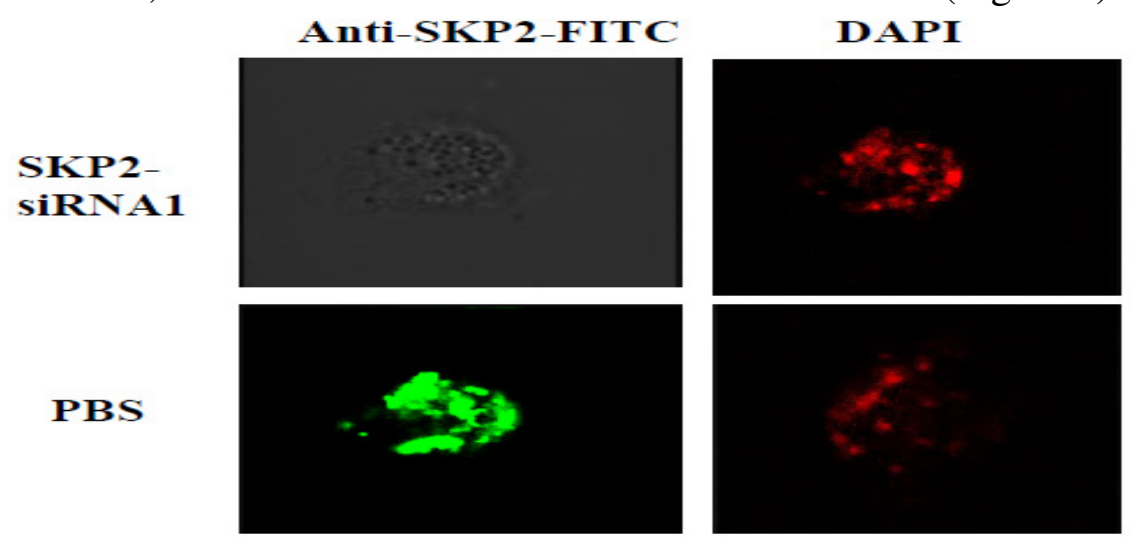

Figure (3): Transfection efficiency

At $48 \mathrm{~h}$ after transfection with SKP2-siRNA1, MCF-7 cells were examined with SKP2 antibody, followed by FITC-conjugated 
secondary antibody, and analysed using fluorescence microscopy. SKP2 antibody bound to the expressed protein (green); nuclei were stained with DAPI (red). The top panel shows SKP2 expression in the cells transfected with SKP2-siRNA1, compared with that in the cells transfected with PBS (bottom panel).
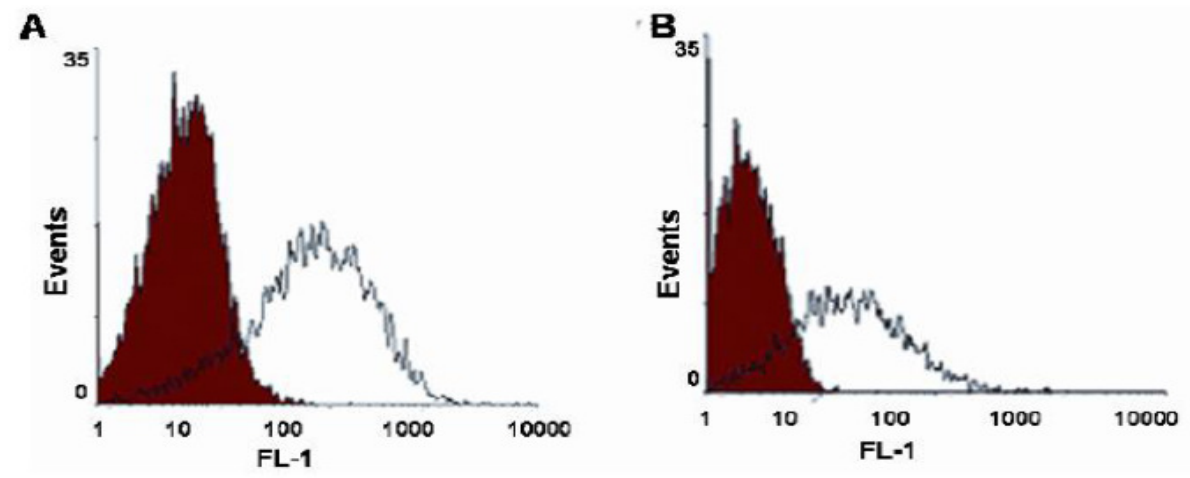

Figure (5): Flow cytometric analysis of cells transfected with FITC-labeled siRNA.

Cells were transfected with $200 \mathrm{nM}$ siRNA. After $12 \mathrm{~h}$, cells were washed twice with 1x PBS and the fluorescence in FL-1 (FITC) was analyzed. (A) MCF-7 cells showed $>95 \%$ transfection efficiency in FL-1. (B) T47D cells showed $>90 \%$ transfection efficiency in FL-1.

\section{DISCUSSION}

Our study revealed the transfection of cancer cell lines with siRNAs targeted against the SKP2 gene can successfully reduce SKP2 gene expression and produce anti-proliferative effects in breast cancer cells. Our results suggest that siRNA is an affect tool on breast cancer cell lines progression. Although two sets of siRNAs targeted to different regions of SKP2 gene, one of the siRNAs had the most potent silencing effect, which was establish with data from previous studies showing that there are variations in the effectiveness of different siRNAs targeting the same gene [11,24]. So the exact site targeted in the RNA is a key factor in silencing the target gene. A mainly interesting finding was the inhibition dose-dependent; simply increasing the siRNA concentration did not enhance inhibition. The stronger inhibitory effect on SKP2 levels depending on the potent 
siRNA, concentration and the combination of different siRNAs could be had additive or synergistic effects. The high concentration of antisense oligonucleotides can inhibit SKP2 expression in breast cancer cells [26], the siRNA effectively reduced the target gene level without causing generalized toxicity, this result suggests that siRNA has evident advantages over an antisense strategy in decreasing gene expression, and therefore this approach has possible application in gene therapy for breast tumors[6, 27]. In this study, the reduction of SKP2 in MCF-7 breast cancer cells was associated with raised p27 expression, which was followed by the induction of apoptosis, decreased cell proliferation, and inhibition of the G1 phase. This supposes that the overexpression of SKP2 is a primary cause of the down regulation of $\mathrm{p} 27$ in certain breast cancers, and provides strong evidence that SKP2 is an oncogene and is functionally activated during the pathogenesis of breast cancer. T47D cells showed no significance with used siRNAs, suggesting that other factors, such as additional procedures or alternative pathways, might be contribute to the down regulation of p27 expression in a subset of tumor cells [5, 9, 14, 28]. Future studies are needed for the demonstration of reduction of SKP2 by siRNAs, because induction breast cancer cell apoptosis followed by the inhibition of cell proliferation. This observation is in agreement with the reports suggesting that the inhibition of SKP2induced apoptosis is characterized by an elevated level of p27, and a decrease in the $\mathrm{G} 1$ phase $[13,16,29]$. The findings of a high level of p27 and low G1 cell population resulting from inhibited SKP2 are also reliable with the function of $\mathrm{p} 27$ as a key regulator of G1 progression[30, 31]. Thus, SKP2-siRNA could inhibit tumor growth by controlling the level of SKP2, which could in turn activate the p27 pathway that leads to apoptosis, in the present study, we found that SKP2 reduction induced increased levels of $\mathrm{p} 27$, followed by depletion of expression of cyclin E and CDK2 in breast cancers [6]. The mechanism underlying this observation may be related to the ability of the SKP2-p27-Cdk1, that control DNA synthesis and mitosis, and p27 is the principal downstream effector of the SKP2 ubiquitin ligases [28, 32]. Low levels of p27 have previously been individually associated with both basal-like breast cancer and a poor outcome after diagnosis [26, 27]. Skp2 inhibitor controls cancer and sensitivity to chemotherapeutic agents in multiple animal tumor models. These results recognize a new novel small molecule that 
targets the Skp2 and reduces tumor growth by inducing cellular apoptosis [20, 33]. CONCLUSION: Our results suggest that siRNAmediated gene silencing of Skp2 can be a potent tool of cancer gene therapy for suppression of Skp2 in breast cancer.

\section{REFERENCES}

1. Brown, L.C., R.W. Mutter, and M.Y. Halyard, Benefits, risks, and safety of external beam radiation therapy for breast cancer. Int $\mathbf{J}$ Womens Health, 2015. 7: p. 449-58.

2. Cahir, C., et al., Women's experiences of hormonal therapy for breast cancer: exploring influences on medication-taking behaviour. Support Care Cancer, 2015.

3. Fujii, T., et al., Preclinical and clinical studies of novel breast cancer drugs targeting molecules involved in protein kinase $C$ signaling, the putative metastasis-suppressor gene Cap43 and the Y-box binding protein-1. Curr Med Chem, 2008. 15(6): p. 528-37.

4. Cheng, H., et al., Skp2 regulates subcellular localization of PPARgamma by MEK signaling pathways in human breast cancer. Int J Mol Sci, 2013. 14(8): p. 16554-69.

5. Masuda, T.A., et al., Clinical and biological significance of $S$ phase kinase-associated protein 2 (Skp2) gene expression in gastric carcinoma: modulation of malignant phenotype by $S \mathrm{kp} 2$ overexpression, possibly via p27 proteolysis. Cancer Res, 2002. 62(13): p. 3819-25.

6. Radke, S., A. Pirkmaier, and D. Germain, Differential expression of the F-box proteins Skp2 and Skp2B in breast cancer. Oncogene, 2005. 24(21): p. 3448-58.

7. Chan, C.H., et al., Skp2: a dream target in the coming age of cancer therapy. Cell Cycle, 2014. 13(5): p. 679-80.

8. Arbini, A.A., et al., Skp2 overexpression is associated with loss of BRCA2 protein in human prostate cancer. Am J Pathol, 2011. 178(5): p. 2367-76.

9. Bochis, O.V., et al., The role of Skp2 and its substrate CDKN1B (p27) in colorectal cancer. J Gastrointestin Liver Dis, 2015. 24(2): p. 225-34.

10. Stewart, S.A., et al., Antimitogenesis linked to regulation of Skp2 gene expression. J Biol Chem, 2004. 279(28): p. 29109-13.

12 
11. Hernandez-Negrete, I., et al., Adhesion-dependent Skp2 transcription requires selenocysteine tRNA gene transcriptionactivating factor (STAF). Biochem J, 2011. 436(1): p. 133-43.

12. Hershko, D.D., Oncogenic properties and prognostic implications of the ubiquitin ligase Skp2 in cancer. Cancer, 2008. 112(7): p. 1415-24.

13. Fujita, T., et al., Dissection of the APCCdh1-Skp2 cascade in breast cancer. Clin Cancer Res, 2008. 14(7): p. 1966-75.

14. Sonoda, H., et al., Significance of skp2 expression in primary breast cancer. Clin Cancer Res, 2006. 12(4): p. 1215-20.

15. Gao, S., et al., miRNA oligonucleotide and sponge for miRNA-21 inhibition mediated by PEI-PLL in breast cancer therapy. Acta Biomater, 2015.

16. Harada, K., et al., Down-regulation of S-phase kinase associated protein 2 (Skp2) induces apoptosis in oral cancer cells. Oral Oncol, 2005. 41(6): p. 623-30.

17. Jiang, K., et al., Targeted delivery of CXCR4-siRNA by scFv for HER2(+) breast cancer therapy. Biomaterials, 2015. 59: p. 77-87.

18. Katagiri, Y., Y. Hozumi, and S. Kondo, Knockdown of Skp2 by siRNA inhibits melanoma cell growth in vitro and in vivo. $\mathrm{J}$ Dermatol Sci, 2006. 42(3): p. 215-24.

19. Kawakami, K., et al., Increased SKP 2 and CKS1 gene expression contributes to the progression of human urothelial carcinoma. $\mathrm{J}$ Urol, 2007. 178(1): p. 301-7.

20. Pascal, L.E. and Z. Wang, Virtual drug design: Skp1-Skp2 inhibition targets cancer stem cells. Asian J Androl, 2013. 15(6): p. 717-8.

21. Bertoli, G., C. Cava, and I. Castiglioni, MicroRNAs: New Biomarkers for Diagnosis, Prognosis, Therapy Prediction and Therapeutic Tools for Breast Cancer. Theranostics, 2015. 5(10): p. $1122-43$.

22. Frescas, D. and M. Pagano, Deregulated proteolysis by the F-box proteins SKP2 and beta-TrCP: tipping the scales of cancer. Nat Rev Cancer, 2008. 8(6): p. 438-49.

23. Chan, C.H., et al., Regulation of Skp2 expression and activity and its role in cancer progression. ScientificWorldJournal, 2010. 10: p. 1001-15. 
24. Chan, C.H., et al., Pharmacological inactivation of Skp2 SCF ubiquitin ligase restricts cancer stem cell traits and cancer progression. Cell, 2013. 154(3): p. 556-68.

25. Tonissi, F., et al., The effect of paclitaxel and nab-paclitaxel in combination with anti-angiogenic therapy in breast cancer cell lines. Invest New Drugs, 2015. 33(4): p. 801-9.

26. Kaboli, P.J., et al., MicroRNA-based therapy and breast cancer: A comprehensive review of novel therapeutic strategies from diagnosis to treatment. Pharmacol Res, 2015. 97: p. 104-21.

27. Voduc, D., et al., The combination of high cyclin E and Skp2 expression in breast cancer is associated with a poor prognosis and the basal phenotype. Hum Pathol, 2008. 39(10): p.1431-7.

28. Traub, F., et al., Prognostic impact of Skp2 and p27 in human breast cancer. Breast Cancer Res Treat, 2006. 99(2): p. 185-91.

29. Schneider, G., et al., IKKalpha controls p52/RelB at the skp2 gene promoter to regulate G1- to $S$-phase progression. EMBO J, 2006. 25(16): p. 3801-12.

30. Xiao, J., et al., SKP2 siRNA inhibits the degradation of P27kipl and down-regulates the expression of MRP in HL-60/A cells. Acta Biochim Biophys Sin (Shanghai), 2009. 41(8): p. 699-708.

31. Haque, I., et al., CCN5/WISP-2 promotes growth arrest of triplenegative breast cancer cells through accumulation and trafficking of p27(Kip1) via Skp2 and FOXO3a regulation. Oncogene, 2015. 34(24): p. 3152-63.

32. Shapira, M., et al., The mTOR inhibitor rapamycin downregulates the expression of the ubiquitin ligase subunit Skp2 in breast cancer cells. Breast Cancer Res, 2006. 8(4): p. R46.

33. Ji, P., et al., Disrupting Skp2-cyclin A interaction with a blocking peptide induces selective cancer cell killing. Mol Cancer Ther, 2007. 6(2): p. 684-91.

\section{declaration:}

References are not following the Journal style . 